CASE REPORT

\author{
M. Dugar \\ A. da Graca Bandeira \\ J. Bruns, Jr \\ P.M. Som
}

\section{Unilateral Hypopharyngitis, Cellulitis, and a Multinodular Goiter: A Triad of Findings Suggestive of Acute Suppurative Thyroiditis}

SUMmARY: Acute suppurative thyroiditis is an uncommon disorder, which has been associated with pre-existing thyroid disease, especially a multinodular goiter. We describe a case of a woman who presented clinically with an acute inflammatory condition of the neck. On CT examination, she had a triad of a multinodular goiter, a unilateral hypopharyngitis, and a surrounding cellulitis. We suggest that the constellation of these imaging findings should alert the radiologist to the diagnosis of acute thyroiditis.
A cute suppurative thyroiditis (AST) is an uncommon inflammatory disease, most often caused by pyogenic bacteria. ${ }^{1-3}$ Pre-existent thyroid disease has been reported in $50 \%-100 \%$ of the cases, most often in a multinodular goiter. ${ }^{1,2}$ A fistula from the apex of the pyriform sinus to the thyroid gland has been identified in a few cases; however, almost all of these cases were in children with a history of recurrent thyroid infections. ${ }^{4}$ To our knowledge, in most cases of acute thyroiditis affecting adults, no such fistulous tract has been reported. In most cases, it is believed that the infection may reach the thyroid gland via a hematogenous route. We present a case of a woman who presented for the first time in an acute condition with a swollen inflammatory neck and who, on CT, had a multinodular goiter, surrounding unilateral cellulitis, and a unilateral hypopharyngitis. It was the constellation of these findings that suggested acute thyroiditis.

\section{Case Report}

The patient was a 38-year-old woman who presented with a 2-week history of progressively worsening dysphonia and dysphagia, with left-ear pain on swallowing. When neck swelling developed, she saw her local physician, who diagnosed an acute pharyngitis and a benign thyroid cyst. She was started on amoxicillin and clavulanic acid (Augmentin) and prednisone, but after 5 days with no improvement, she presented to our ambulatory emergency department, at which time she could not swallow solid food. There was no history of trauma, neurologic complaint, vomiting, or shortness of breath. No signs or symptoms of thyroid storm or hyperthyroidism were present.

Her vital signs were normal. There was visible and palpable swelling over the left anterolateral neck, and the left lateral nodes were tender and enlarged. Findings of the remainder of her physical examination were normal. The initial clinical assessment resulted in a differential diagnosis that included a goiter with possible intraglandular hemorrhage and lymphadenopathy, an infected branchial cleft or thyroglossal duct cyst, and pharyngitis with a possible neck abscess. The patient was treated in the emergency

Received March 3, 2009; accepted after revision March 22.

From the Departments of Radiology (M.D., P.M.S.) and Emergency Medicine (J.B.), Mount Sinai Medical Center, New York, NY; and Department of Radiology (A.d.G.B.), Centro Hospitalar de Lisboa Central, Lisbon, Portugal.

Please address correspondence to Peter M. Som, MD, FACR, Department of Radiology, Mount Sinai Medical Center, Box 1234, One Gustave L. Levy PI, New York, NY 10029-6574; e-mail: Peter.Som@MSSM.Edu

DOI 10.3174/ajnr.A1642 department with dexamethasone $10 \mathrm{mg}$ intravenously every 6 hours and antibiotics for typical oropharyngeal bacterial pathogens as well as Streptococcus and odontogenic anaerobes. Neck pain was formidable and managed with ketarolac (Toradol), oxycodone hydrochloride with acetaminophen (Percocet), and morphine as needed. Her pain level was 10/10 (1 lowest, 10 highest) at initial triage but declined to a maximum of $3 / 10$ during the next 12 hours. Her laboratory values were within normal limits.

A chest radiograph showed a superior mediastinal mass causing right tracheal deviation thought to be due to a substernal thyroid. A contrast-enhanced CT scan of the neck showed edematous swelling of the left hypopharynx and a supraglottic larynx. Cellulitic changes extended caudally in the left neck to surround a multinodular goiter (Fig. 1). There was no airway compromise. These imaging findings suggested that the diagnosis could be an acute thyroiditis.

She was transferred to the ear, nose, and throat service and maintained on intravenous hydration, pain management, steroids, and antibiotics. Results of a sonography-guided fine-needle aspiration of the thyroid gland were consistent with a goiter with cystic degeneration; no malignant cells were present. No fistula was identified on the sonographic examination. Endoscopy also failed to reveal a pyriform sinus fistula. She was discharged after 1 week to be followed by her local physician and an endocrinologist for thyroid-function monitoring. Her discharge medications included narcotic analgesics as needed, methylprednisolone (Solu-Medrol) dose pack, and levofloxacin (Levaquin) $500 \mathrm{mg}$ daily for 10 days.

She remained symptom-free except for the goitrous mass in her neck, and 4 months later, she was readmitted to the hospital for a thyroidectomy. At this time, a barium swallow was performed (Fig 2 ), which showed no evidence of a fistula. At surgery, her goiter was removed, and again no fistulous tract was identified.

\section{Discussion}

In general, the thyroid gland has a relatively high resistance to infection. Multiple factors contribute to this resistance, including the protective fibrous capsule of the thyroid, an extensive venous and lymphatic drainage, and a high concentration of iodine. ${ }^{5,6}$ On the other hand, the highly vascularized nature of the thyroid gland can lead to its seeding from hematogenous sources.

Despite this potential hematogenous spread of infection, AST remains a relatively uncommon disorder. Although it is often secondary to a pyogenic bacterial infection, it can rarely be secondary to a fungal, parasitic, or viral infection or can be caused by Pneumocystis carinii, especially in immunosup- 

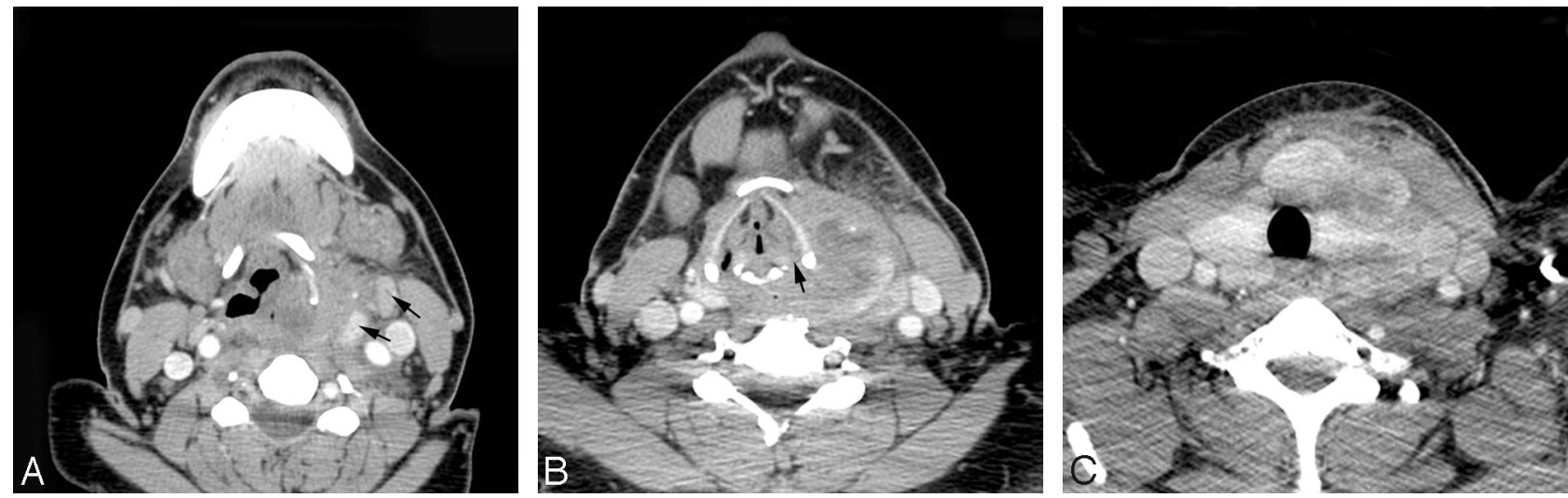

Fig 1. Serial axial CT scans from a cranial to caudal direction. A, Swelling of the left hypopharynx and left aryepiglottic fold with infiltration of the adjacent left-neck fat planes. The uppe edges of the displaced thyroid gland (arrows) can be seen. B, Extension of the slightly low-attenuation edematous disease caudally in the neck. The hypopharynx and thyroid gland are

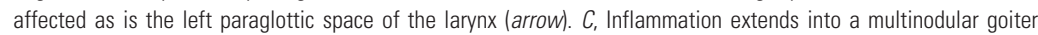
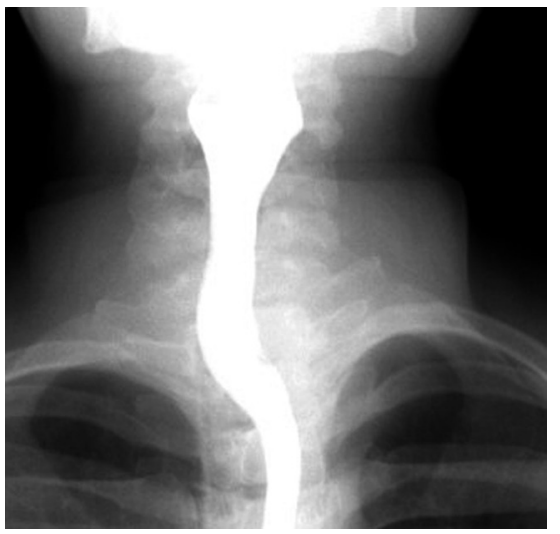

Fig 2. Frontal spot film of a barium swallow shows a normal anatomy of the pharynx and the upper esophagus with no evidence of a fistula.

pressed or immunocompromised patients. ${ }^{3,6-8}$ A study from Nigeria of 84 women with endemic goiters demonstrated that acute thyroiditis occurred in $10.7 \%$ of the patients. In this study, the most common causative agent was Staphylococcus aureus. Less often Streptococcus pyogenes and Pseudomonas organisms were the offending agents. ${ }^{1}$

The most common route of spread is very likely hematogenous from a pharyngitis. However, a few cases have been reported with a congenital fistula to the thyroid gland from the apex of the pyriform sinus. When this occurs, the fistula likely represents a rare fourth branchial anomaly, and these reported cases share certain findings in common, including onset in infancy or childhood, left thyroid lobe involvement, and frequent recurrences unless surgery is performed. ${ }^{4}$ Because our patient developed her thyroiditis as an adult, had no prior history of such episodes, and has had no subsequent episodes, it is unlikely that she had such a rare fistula. Furthermore, no fistula was identified on CT, sonography, barium swallow, or at surgery.

As mentioned, in most cases of AST, the patient has an underlying thyroid condition, such as a multinodular goiter. However, chronic thyroiditis, an adenoma, a carcinoma, or a history of direct thyroid trauma has also been reported. ${ }^{6}$

The typical clinical presentation of AST is swelling and tenderness in the anterior neck, which may be accompanied by fever, dysphagia, hoarseness, or coexisting pharyngitis. ${ }^{9,10}$ Laboratory findings characteristically include an elevated erythrocyte sedimentation rate and leukocytosis. Results of thyroid function tests are generally normal. ${ }^{6,8-10}$

Thyroid sonography usually demonstrates a thyroid gland with diffusely decreased echogenicity intermixed with hyperechoic trabeculae. Sonography is useful for detecting a local abscess, performing drainage of the abscess, and obtaining a specimen to identify the causative pathogen. Contrast-enhanced CT also provides excellent assessment of the thyroid gland, the pharynx, and adjacent soft tissues as well as the presence of an abscess. ${ }^{6,11} \mathrm{MR}$ imaging, although not performed in our patient, can also be useful for depicting an abscess; it would typically demonstrate restricted diffusion on diffusion-weighted imaging. ${ }^{12}$ Antibiotic therapy is typically curative. Although in almost all adult cases, a fistula is not identified, if a fistula is present, surgical resection is suggested. If an abscess is present, surgical incision and drainage may be necessary. ${ }^{1}$

This case demonstrates that when the triad of a multinodular goiter and a unilateral hypopharyngitis with a surrounding cellulitis is present, the diagnosis of acute thyroiditis should be considered by the radiologist, because this diagnosis may not be initially considered purely on the basis of the clinical presentation. Although in children AST may be associated with a fistula, in adults such a fistula is rarely reported, and the spread of infection from the pharynx to the thyroid is believed to be via a hematogenous route.

\section{References}

1. Ameh EA, Sabo SY, Nmadu PT. The risk of infective thyroiditis in nodular goiters. East Afr Med J 1998;75:425-27

2. Gnepp DR. Pathology of the thyroid and parathyroid glands. In: Gnepp DR ed. Diagnostic Surgical Pathology of the Head and Neck. Philadelphia: WB Saunders Company; 2001:441-42

3. Singer PA. Thyroiditis: acute, subacute, and chronic. Med Clin North Am 1991;75:61-77

4. Miyauchi A, Matsuzuka F, Takai S, et al. Piriform sinus fistula. Arch Surg 1981;116:66-69

5. Farwell AP. Acute infectious thyroiditis. In: Braverman LE, Utiger RD, eds. Werner and Ingbar's The Thyroid: A Fundamental and Clinical Text. 9th ed. Philadelphia: Lippincott Williams \& Wilkins; 2004:541-47

6. Shah SS, Baum SG. Diagnosis and management of infectious thyroiditis. Cur Infect Dis Rep 2000;2:147-53 
7. Guttler R, Singer PA, Axline SG, et al. Pneumocystis carinii thyroiditis: report of three cases and review of the literature. Arch Intern Med 1993;153:393-96

8. Yu EH, Ko WC, Chuang YC, et al. Suppurative Acinetobacter baumanii thyroiditis with bacteremic pneumonia: case report and review. Clin Infect Dis 1998;27:1286-90

9. Berger SA, Zonszein J, Villamena P, et al. Infectious diseases of the thyroid gland. Rev Infect Dis 1983;5:108-22
10. Hay ID. Thyroiditis: a clinical update. Mayo Clin Proc 1985;60:836-43

11. Singh SK, Agrawal DM, Kumar M, et al. Fine-needle aspiration cytology in the management of acute suppurative thyroiditis. Ear Nose Throat J 1994;73:415-17

12. Koc O, Paksoy Y, Erayman I, et al. Role of diffusion weighted MR in the discrimination diagnosis of the cystic and/or necrotic head and neck lesions. Eur J Radiol 2007;62:205-13. Epub 2006 Dec 22 